\title{
Fingerprint Recognition and its Advanced Features
}

\author{
Soukhya S M, Sonu G, L Karthik Narayan \\ Dr.Manju VC (Prof, Department of TCE, KSIT) \\ Department of Telecommunication, K S Institute of Technology, Bangalore, India
}

\begin{abstract}
Fingerprint recognition is one of the most popular and successful methods used for person identification, which takes advantage of the fact that the fingerprint has some unique characteristics called minutiae; which are points where a curve track finishes, intersect with other track or branches off. Biometric identification systems using fingerprints patterns are called AFIS (Automatic Fingerprint Identification System). Fingerprint Recognition is one of the research hot spots in Biometric. It refers to the automated method of verifying a match between two human
\end{abstract}

fingerprints. It is essentially a challenging pattern recognition problem where two competing error rates: the False Accept Rate (FAR) and the False Reject Rate (FRR) need to be minimized. Advancement of computing capabilities led to the development of Automated Fingerprint Authentication Systems (AFIS) and this led to extensive research especially in the last two decades. In this paper, we attempt to give a comprehensive scoping of the fingerprint recognition problem and address its major design and implementation issues as well as give an insight into its future prospects.

Keywords: - Fingerprint, Pre-processing, Feature extraction, Matching, Databases .

\section{INTRODUCTION}

Along of various biometrics techniques, In the past few decades human-beings have been addicted to various technologies such as captured photos, scanned signatures, bar code systems, verification Id \& so on. Also, Biometrics is one of the applications in Image processing which refers to technologies that used physiological or behavioral characteristics of human body for the user authentication. The biometric authentication system based on two modes: Enrolment and Recognition. In the enrolment mode, the biometric data is acquired from the sensor and stored in a database along with the person's identity for the recognition. In the recognition mode, the biometric data is re-acquired from the sensor and compared to the stored data to determine the user identity. Biometric recognition based on uniqueness and permanence. The uniqueness means that there is no similarity of feature between two different biometrics data. For example, there are no two humans having the same fingerprint feature even if they are twins. And when the features of biometrics do not change over the lifetime or aging, it is called permanence. Biometrics can have physiological or behavioral characteristics. The physiological characteristics are included in the physical part of body such as (fingerprint, palm print, iris, face, DNA, hand geometry, retina... etc).
The behavioral characteristics are based on an action taken by a person such as (Voice recognition, keystroke-scan, and signature-scan). Any biometrics system including two phases first phase is enrollment phase and second is recognition phase. The recognition phase divided to two things which is verification and identification. During the enrollment phase the biometrics data are captured and generate digital image then Pre-processingapply to digital image for removing unwanted data and apply the postprocessing than store this data in database.In the case of identification process the fingerprint acquired from one person is compared with all the fingerprints which store in database. Also it is known as $(1: \mathrm{N})$ matching. it is used in the process of seeking the criminals.In the verification process the person's fingerprint is verified from the database by using matching algorithms. Also it is known as (1:1) Matching. It is the comparison of a claimant fingerprint against enroll fingerprint, initially the person enrolls his/her fingerprint into verification system, and the result show whether the fingerprint which take from the user is matching with the fingerprint store as a template in database or not match. The Enrollment, Identification and Verification process shown in the Fig.1.Process of Enrollment, identification and verification The fingertip surface is a combination of many of ridges and many of valleys.

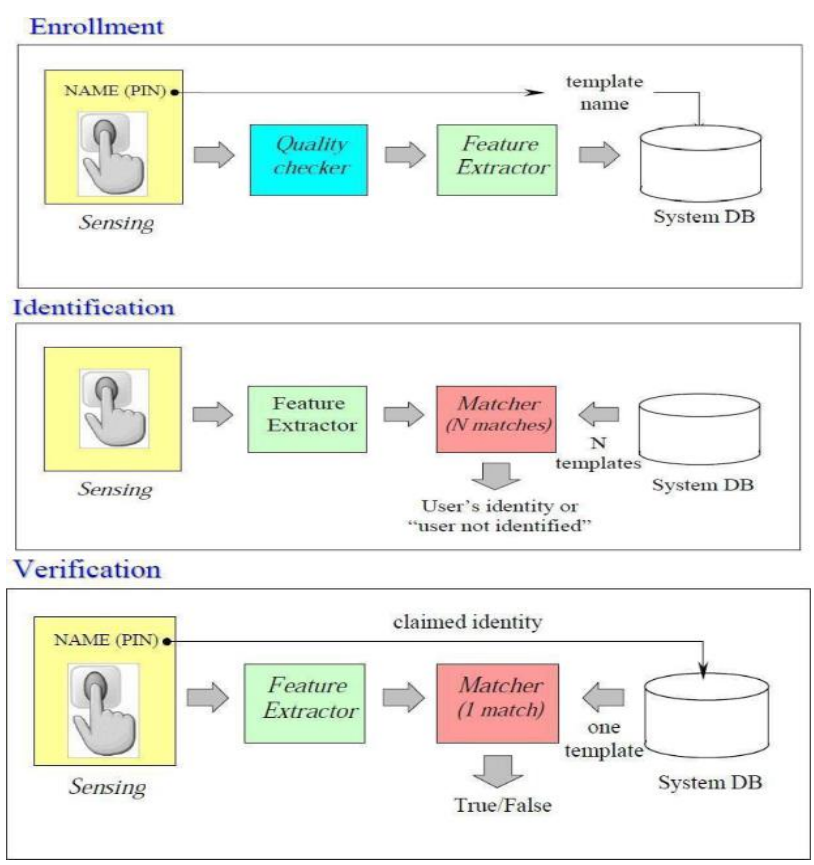

Figure 1: Process of Enrollment, identification and verification 
The fingertip surface is a combination of many of ridges and many of valleys. In case of the ridge which declare as black lines and the valleys declare as white lines are show in Fig.2. The minutiae points are the points where the ridge structure changes such as bifurcation and end point.

Now a day old security methods password is not accurate and outstanding to protect individual belongings therefor for this purpose used biometrics recognition system.

\section{FINGERPRINT}

Fingerprints are graphical patterns of ridges and valleys on the surface of fingertips, the ridge ending and ridge bifurcation is called minutiae as shown in fig.3. There are many methods based on minutiae-based fingerprint representation were proposed in [1], [2]. Every person has a unique fingerprint from any other person. The fingerprint identification is based on two basic assumptions: - In variance and Singularity In variance: means the fingerprint characteristics do not change along the life. Singularity: means the fingerprint is unique and no two persons have the same pattern of fingerprint.

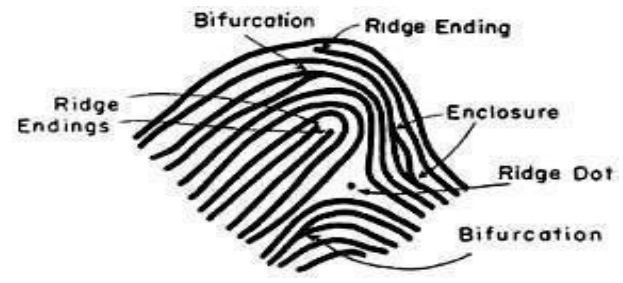

FIGURE 2: Different ridge features on fingerprint image

Table I. shows the terms and definitions of fingerprint structure

\begin{tabular}{|r|l|l|}
\hline $\begin{array}{r}N \\
o\end{array}$ & \multicolumn{1}{|c|}{ Term } & \\
\hline 1 & Termination & The location where a ridge comes to an end. \\
\hline 2 & Bifurcation & $\begin{array}{l}\text { The location where a ridge divides into two } \\
\text { separate ridges. }\end{array}$ \\
\hline 3 & Binarization & $\begin{array}{l}\text { a process to transform the image from 256 levels } \\
\text { to two levels(0,1)refers to (black and white) } \\
\text { respectively }\end{array}$ \\
\hline 4 & Thinning & $\begin{array}{l}\text { The process of reducing the width of each ridge } \\
\text { to one pixel . }\end{array}$ \\
\hline 5 & $\begin{array}{l}\text { Termination } \\
\text { Angle }\end{array}$ & $\begin{array}{l}\text { Considered as angle between the direction of the } \\
\text { ridge and the horizontal }\end{array}$ \\
\hline 6 & $\begin{array}{l}\text { Bifurcation } \\
\text { Angle }\end{array}$ & $\begin{array}{l}\text { Which Considered as the angle between the } \\
\text { direction of the valley ending between the } \\
\text { bifurcations and the horizontal. }\end{array}$ \\
\hline 7 & Matching Score & $\begin{array}{l}\text { It is the mesure of similarity between the input } \\
\text { and template data }\end{array}$ \\
\hline 8 & $\begin{array}{l}\text { False Non } \\
\text { Matching Ratio }\end{array}$ & $\begin{array}{l}\text { The system fail to detect a match between input } \\
\text { and template in database } .\end{array}$ \\
\hline
\end{tabular}

\section{FINGERPRINT RECOGNITION SYSTEM}

Now a day old security methods password is not accurate and outstanding to protect individual belongings therefor for this purpose used biometrics recognition system. Fingerprints is a consolidation of many ridges and many valleys on the surface of fingerprints ridges are declare as black lines shown in Figure

1 and valley declare as white lines [27-30]. Biometrics of human is splatted into two categories 1 behavior of biometrics 2 physiological biometrics. Every human has its own biometrics characteristics and can be identifying through these characteristics. Fingerprints biometrics system has four basic process or internships that are: collection, extraction, comparison decision or validation or identification fingerprints recognition system (FRS) requires a comparison or match of his/her fingerprints with the fingerprints in the database to indicate individuals in the storage [30]. Another major purpose of the system is to be used as a security device [31]. In 1899 galton first time explored the feasibility of using fingerprints Recognition system for identifying newborn children it was also possible to use fingerprints to recognize children of 2.5 year age Automated fingerprints identification system (AFIS) are widely used method for authentication (1:1 match) or identification and duplication 1: $\mathrm{N}$ search of individuals 1 and now prevalent around the globe providing and accurate. In modern society fingerprints recognition system is the ability to reliably identify individuals'. A much important application like computer security, financial transaction international border crossing and forensics.
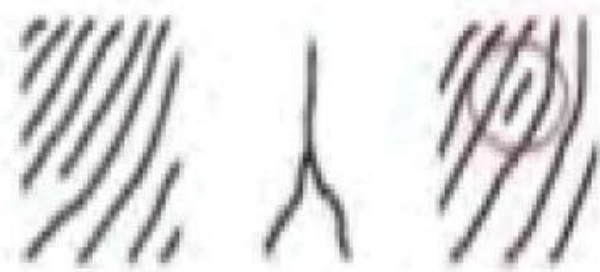

FIGURE 3 :Graphical of ridge and valleys Ridge Ending, Bifurcation and short Ridge [14]

\section{A. WORKING ANDFEATURE}

Fingerprints recognition system is a widely used application and becomes popular and update by the time because manually checking the identity of a person. In the market many techniques are introduced for the recognition with the perspective of human behavior. Biometrics gives us high security that is the reason persistently used for many years and this technique is very simple and easy to use. Biometrics is considering a powerful approach because our ridge and valley surface of fingerprints cannot change and remain the same through a lifetime. Fingerprint recognition is being used for the identification at the several departments as the system security is considered as 
the most important factor at any department of information and technology. Initially the user gets register with the system in which the user will provide the basic and personal information which will be required by the system and also the user will provide the thumb impression to the hardware interface which will store the finger print image as well the other information about the user into the database. After the procedure of the registration, the user will only put the finger at the hardware interface; the system will allow the user to get logged in to the system (Figure 4).

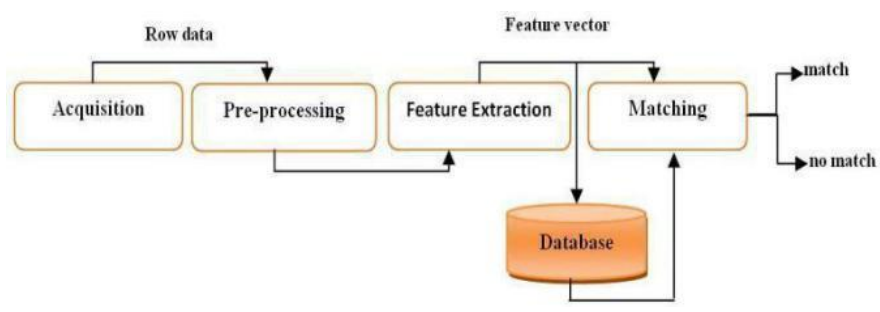

FIGURE 4:Complete process of finger recognition

\section{B. STEPS FOR RECOGNIZING A IMAGE}

Capture image/Image acquisition: It's a very first step, in this stage capture the fingerprint through digital sensors look very blur and noisy because the quality of image is not upright [30].The Image Acquisition stage is the process to obtain images by different ways. There are two ways to capture fingerprint image; online and offline. In the online fingerprint identification the optical fingerprint reader is used to capture the image of fingerprint. The size of fingerprint image will be $260 * 300$ pixels. The offline fingerprint identification is obtained by ink in the area of finger and then put a sheet of white paper on the fingerprint and finally scans the paper to get a digital image.

Pre-processing: In the second step make the quality of image better by applying the worth full algorithm, which remove all the damage area appear on the fingerprint such as noisy, missing minutiae, blur etc. and convert it into a high quality image. In high quality image ridge and valleys are properly recognize [30]. The pre-processing stage is the process of removing unwanted data in the fingerprint image such as noise, reflection .etc. The fingerprint image pre-processing is used to increase the clarity of ridge structure.Yun and Cho [6] in this work they adaptive preprocessing method on fingerprint image and extracts five features from it. and they used clustering method for analyses image quality, and enhance it by their characteristics. This is what pre-processing is performed after distinguishing the fingerprint image quality related to its characteristics.

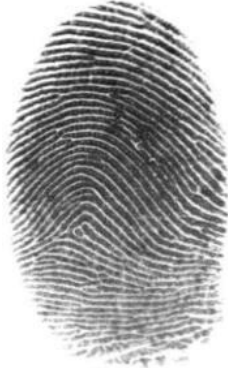

(a)

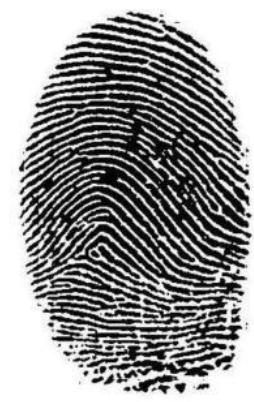

(b)

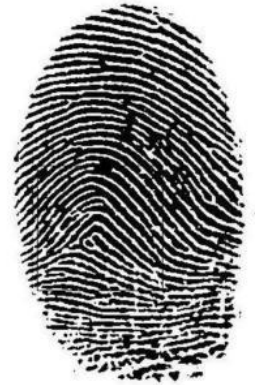

FIGURE 5: Pre-processing stages (a) Original image (b) Enhancement ( c ) Binary image

Feature extraction:The feature extraction process of fingerprint image applied on the output of pre-processing stage. The process of feature extraction depends on set of algorithms. A fingerprint feature extraction program is to locate, measure and encode ridge endings and bifurcations in the fingerprint. There are various methods for extracting the features from the fingerprint image. The famous methods is minutiae extraction algorithm which is find the minutiae points and map their relative placement on the fingerprint .There are two types of minutiae points; Ridge ending and Ridge bifurcation [7]. In[8]

they are used an advanced method for extract feature from fingerprint which done by extract minutiaedirectly from original gray-level images without use binarization and thinning and they use gabor filter methods to extract features from fingerprint.

In this step identify the different features of fingerprint. Feature Extraction relies on three levels such as Level 1: Global Level (identify the delta, whorl, and loop), Level 2: Local Level (check the out of order ridges in most minutiae form. Level 2 worked on Bifurcation, ridge ending, Lake, and Crossover). Level 3: Very Fine Level (work on sweet and white pores detected) [30].

Pattern Recognition (PR): In fourth step firstly divide PR into two categories (i) Decision Theoretic: In this step quantitative descriptor work with patterns such as texture, area, length. (ii) Structural: Relational descriptor describe by qualitative descriptor that also focus on patterns [30] (Figure 6).

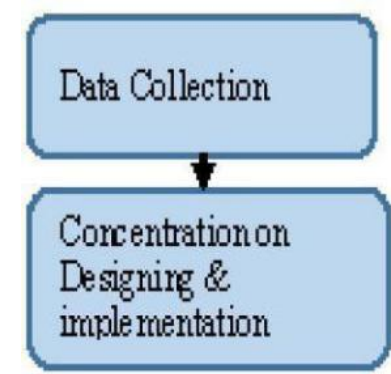

FIGURE 6 :Steps of finger recognition system

Most of the enterprises are using the services of fingerprint recognition for the login purpose because the assigning of privileges was a bit difficult in previous technologies. The 
fingerprint recognition services enables the multiple enterprises where the entrepreneurs can integrate the proper privileges of the system. According to the latest researches, the voting systems should be implemented using the algorithms of finger prints which are less costly as there are a lot of issues about the riggings in the general elections of several countries, so the integration E-voting systems should be most optimized as well as the integrated system should also be based on the correct identification which should be considered as the future work in the field of internet of things and system security (Figure7).

Matching stage:- The matching stage is the process to compare the acquired feature with the template in the database. In other words the process of matching stage is to calculate the degree of similarity between the input test image (for user when he wants to prove his/her identity) and a training image from database (the template which created at the time of enrolment). Matching can be done in three methods: hierarchical approach, classification approach and Coding approaches. The hierarchical approach is increases matching speed at the cost of accuracy [14].Classification approaches assign a class to each biometric in a database. There are many classification methods including KNN classifier [15].

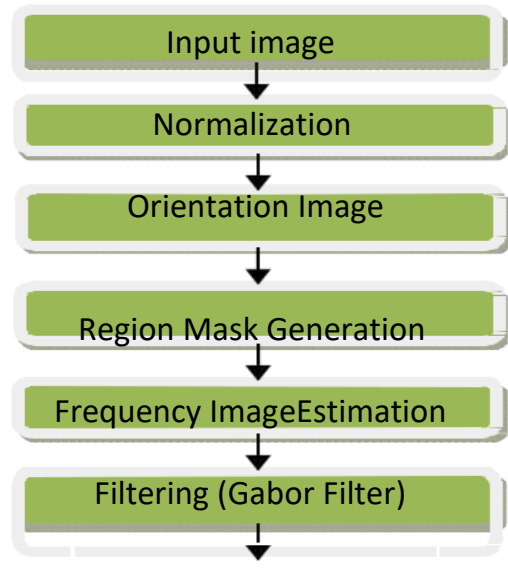

FIGURE 7 :Finger Recognition System

\section{ADVANTAGES OF FINGERPRINT IDENTIFICATION:}
a) It is highly accurate
b) It is unique and can never be same for twopersons.
c) It is the most economical technique.
d) It is easy to use
e) Use of small storage space

\section{APPLICATIONS OF FINGERPRINT IDENTIFICATION:}

a. To identify criminals in crime scenes. It was one of major reasons for development of this technology by FBI in USA.

b. To identify members of an organization. It helps improves security such that only authenticated persons can enter the secured area and not any other members.

c. In Grocery stores to automatically recognize and bill a registered user's credit card or debit card.

\section{CONCLUSION}

This paper give us an overview of fingerprints recognition system Fingerprints is very accurate and reliable technique. Fingerprints recognition system widely used in identification tool and biometrics applications. Biometrics give us high security that is the reason persistently used for many years. We briefly describe the method, approaches and algorithm (indexing algorithm used for efficient search of growing size of fingerprints of fingerprints recognition system. We also discussed the steps metropolitan police started the use of biometrics for identification In 1901 in the UK first stage is acquisition stage or capture, preprocessing (remove the noisy and unwanted data) feature extraction and matching stage for fingerprints recognition purpose with the help of some previous research in detail. In addition we highlighted some security issue which occur in fingerprints approach like spoof attack for this purpose we used fingerprint domain knowledge by extracting local patches centered and aligned using minutiae approach. This approach is able to achieve the significant results. Some basics factors also affecting the fingerprints like age and gender. Finally, the above discussion and result indicate that Fingerprints Recognition System (FRS) is very good accurate efficient and easy to use technique some problem may occur but these problem can be overcome with the passage oftime.

\section{REFRENCES}

[1] N. K. Ratha, K. Karu, S. Chen, and A. K. Jain ," A real-time matching

[2] system for large fingerprint databases ," IEEE Trans.Pattern Anal.Mach Intell.,vol.18,no.8.pp 779813,Aug. 1996

[3] A. K. Jain and L.Hong,"Online fingerprint verification,"IEEE

[4] Trans.Pattern Anal.Mach Intell.,vol.19,no.4.pp 302341,Apr.1997.

[5] Kumar, A., Zhang, D.," Combining fingerprint, palmprint and handyshape for user authentication. In Pattern Recognition, ICPR 2006. 18th International Conference on (Vol. 4, pp. 549-552). IEEE. 2006.

[6] Zhao, F., Tang, X. 2007. Preprocessing and post processing for skeleton-based fingerprint minutiae extraction. Pattern Recognition 40 (2007) 1270 - 1281.

[7] Jiao Ruili and Fan Jing, "VC5509A Based Fingerprint Identification Preprocessing System," International Conference on Signal Processing, pp. 2859 - 2863, 2008.

[8] E. K. Yun and S. B. Cho, "Adaptive Fingerprint Image Enhancement with Fingerprint Image Quality Analysis," International conference of Image and Vision Computing, pp. 101-110, 2006. 
[9] Deshpande, A., S., Patil, S., M., Lathi, R.”A Multimodel Biometric Recognition System based on Fusion of Palmprin Fingerprint and Face". International Journal of Electronics and Computer Science Engineering. ISSN-2277-1956,2012

[10] Zhao, F., Tang, X,"Preprocessing and postprocessing for skeletonybased fingerprint minutiae extraction".Pattern Recognition, 40(4), 1270-1281,2007.

[11] Jain, A. K., Prabhakar, S.,Hong,L."A multichannel approach to fingerprint classification". Pattern Analysis and Machine Intelligence, IEEE Transactions on, 21(4), 348-359.1999.

[12] F. A. Afsar, M. Arif and M. Hussain, "Fingerprint Identification and Verification System using Minutiae Matching," National Conference on Emerging Technologies, pp.141-146, 2004

[13] K.Zebbiche and F.Khelifi,"Region-Based Watermarking of Biometrics Images: Case Study in Fingerprint Images," Proceedings of International Journal of Digital Multimedia Broadcasting, pp. 1-13, 2008.

[14] Yi Chen and A K Jain, "Beyond Minutiae: A Fingerprint Individuality Model with Patteren, Ridge and Pore Features," International Conference on Biometrics, pp. 523-533,2009.

[15] S Tachaphetpiboont and T Amornraksa, "Applying FFT Features for Fingerprint Matching," Proceedings of the IEEE Conference on Wireless Pervasive Computing, pp. 1-5,2006.

[16] J. You, W.K. Kong, D. Zhang, K.H. Cheung, "On hierarchical palmprint coding with multiple features for personal identification in large databases", IEEE Transactions on Circuits and Systems for Video Technology 14 (2) (2004) 234-243, 2004

[17] Gayathri, R. Ramamoorthy, P."Fingerprint and palmprint Recognition Approach based on Multiple Feature extraction". European Journal of scientific research. Vol 76, No 4, 2012.

[18] Arun Ross, Anil Jain and James Reisman, "A Hybrid Fingerprint Matcher," Proceedings of International Conference on Pattern Recognition, pp .1661-1673,2003.

[19] Jong KU Kin, Seung-Hoon Chae, Sung Jin Lim and Sung Bum Pan, "A Study on the Performance Analysis of Hybrid Fingerprint Matching Methods," International Journal of Future Generation Communication and Networking, pp. 23-28,2008

[20] Swapnali Mahadik, K Narayanan, D V Bhoir and Darshana Shah, "Access Control System using Fingerprint Recognition," International Conference on Advances in Computing, Communication and Control, pp. 306-311, 2009.

[21] A K Jain, S Prabhakar and A Chen, "Combining Multiple Matchers for a High Security Fingerprint Verification System," Pattern Recognition Letters, Elsevier Science Direct, vol. 20, pp. 1371- 1379, 1999.

[22] A N Marana and A K Jain, "Ridge-Based Fingerprint Matching using Hough Transform," Proceedings of the IEEE Brazilliab Symposium on Computer Graphica and Image Processing, pp. 112-119, 2005.

[23] Jinwei Gu,"A model-based method for the computation of fingerprints' orientation field ", IEEE Transactions on Image Processing, Volume: 13, No.6, Pages: 821 - 835, DOI: 10.1109/TIP.2003.822608, 2004

[24] JuchengYang; jinWook Shin; BungJun Min; JongBin Park; Dongsun Park," Fingerprint Matching Using Invariant Moment

[25] FingerCode and Learning Vector Quantization Neural Network" , IEEE Conference Publications on Computational

[26] Intelligence and Security, Volume: 1, Pages: 735 - 738, DOI: 10.1109/ICCIAS.2006.294231, 2006.

[27] WangYuan,YaoLixiu,ZhouFuqiang,“A Real Time Fingerprint Recognition System Based On Novel Fingerprint Matching Strategy", 8th International Conference on Electronic Measurement and Instruments. ICEMI '07. Pages: 1-81 - 1-85, DOI: 10.1109/ICEMI.2007.4350576, 2007.

[28] Abrishambaf,R.Demirel,Hasan,Kale,I,"A fully CNN based fingerprint recognition system", 11th International Workshop on Cellular Neural Networks and Their Applications. CNNA 2008. IEEE Conference Publications,Pages: 146 - 149, DOI: 10.1109/CNNA.2008.4588667, 2008 .

[29] Kumar,R. Chandra,P. Hanmandlu, M.,'Local directional pattern (LDP) based fingerprint matching using SLFNN", IEEE Second International Conference on Image Information

[30] Processing (ICIIP), Pages: 493 - 498, DOI: 10.1109/ICIIP.2013.6707640, 2013.
[31] Jucheng Yang,Shanjuan Xie,Sook Yoon, Dongsun Park, Zhijun Fang, Shouyuan Yang,"Fingerprint matching based on extreme learning

[32] Mieee MSA, Muhamad HA (2017) Effective fingerprint recognition approach based on double fingerprint thumb, pp: 7580.

[33] Ahmad AS, Hassan R, Ibrahim N (2017) An investigation

[34] of matching approaches in fingerprints Identification, pp: 9-15. [29]Willis AJ, Myers L (2001) A cost effective fingerprint recognition system for use with low-quality prints and damaged fingertips. Pattern Recognition 34: 255-270.

[35] [30]Ali MMH (2016) Fingerprint Recognition for Person Identification and Verification Based on Minutiae Matching. [31]Cao K, Jain AK (2017) Fingerprint Indexing and Matching: An Integrated Approach. pp: 437-445.

[36] Chugh T, Cao K, Jain AK (2018) Fingerprint Spoof Buster: Use of Minutiae-centered Patches. IEEE Transactions on Information Forensics and Security 13: 131-138.

[37] Jain AK, Arora SS, Cao K (2016) Fingerprint Recognition of Young Children. IEEE Transactions on Information Forensics and Security 60: 1-14.

[38] Wang J, Wei T, Li X, Zhang B (2014 Near-Infrared-LightMediated Imaging of Latent Fingerprints based on Molecular Recognition. Applied Chemistry 53: 1642- 1646.

[39] Cao K, Jain AK (2015) Learning Fingerprint Reconstruction From Minutiae to Image. IEEE Transactions on Information Forensics and Security 10: 104-117.

[40] Engelsma JJ, Cao K (2016) Fingerprint Match in Box. [37]Engelsma JJ, Cao K, Jain AK (2012) RaspiReader: Open Source Fingerprint Reader. pp: 1-14.

[41] Jain AK, Nandakumar K, Ross A (2016) 50 years of biometric research: Accomplishments, challenges, and opportunities. Pattern Recognition Letters, pp: 1-26.

[42] Montesanto,A.Baldassarri,P.Vallesi,G.Tascini,G.," Fingerprints Recognition Using Minutiae Extraction:aFuzzy Approach.",IEEE Conference Publications,14th International Conference on Image Analysis and Processing, ICIAP 2007, Pages: 229 - 234, DOI: 10.1109/ICIAP.2007.4362784, 2007.

[43] Morteza Zahedi, Ozra Rostami Ghadi ," Combining Gabor filter and FFT for fingerprint enhancement based on a regional adaption method and automatic segmentation",springer verlag london 2013,SIViP,DOI 10.1007/s11760-013-0436-3, 2013

[44] Wilson CL, Candela GT, Grother PJ, Watson CI, Wilkinson RA " Massively parallel neural network fingerprint classification system. National Institute of Standards and Technology; NISTIR 4880. 1992

[45] Karu K, Jain A ” Fingerprint classification” . Patt Recog 29(3):389- 404, 1996 [31] Senior A ,"A hidden Markov model fingerprint classifier". Conference record of the Thirty-First

[46] Asilomar Conference on Signals, Systems \& Computers 1:306310,1997

[47] Hong L, Jain ." Classification of fingerprint images". In: Proceedings of the 11th Scandinavian Conference on Image Analysis, Kangerlussuaq, Greenland, June 1999

[48] Yao Y, Frasconi P, Pontil M, "Fingerprint classification

[49] with combinations of support vector machines" . In: Proceedings of the 3rd International Conference on Audio and Video Based Biometric Person Authentication, Halmstad, Sweden, June 2001

[50] Chang J, Fan K, " A new model for fingerprint classification by ridge distribution sequences". Patt Recog 35(6):1209-1223 , 2002

[51] Yao Y, Marcialis G, Pontil M, Frasconi P, Roli F , Combining flat and structured representations for fingerprint classification with recursive neural networks and support vector machines" Patt Recog 36(2):397-406, 2003. 